\title{
On-Chip Actuation of an In-Plane Compliant Bistable Micromechanism
}

\author{
Michael S. Baker \\ Larry L. Howell \\ Ihowell@byu.edu
}

Follow this and additional works at: https://scholarsarchive.byu.edu/facpub

Part of the Mechanical Engineering Commons

\section{Original Publication Citation}

Baker, M.S. and Howell, L.L., â€œOn-Chip Actuation of an In-Plane Compliant Bistable Micromechanism,â€ Journal of Microelectromechanical Systems, Trans. IEEE and ASME, Vol. 11, No. 5, pp. 566-573, 22.

\section{BYU ScholarsArchive Citation}

Baker, Michael S. and Howell, Larry L., "On-Chip Actuation of an In-Plane Compliant Bistable Micromechanism" (2002). Faculty Publications. 519.

https://scholarsarchive.byu.edu/facpub/519 


\title{
On-Chip Actuation of an In-Plane Compliant Bistable Micromechanism
}

\author{
Michael S. Baker and Larry L. Howell
}

\begin{abstract}
A compliant bistable micromechanism has been developed which can be switched in either direction using on-chip thermal actuation. The energy storage and bistable behavior of the mechanism is achieved through the elastic deflection of compliant segments. The Pseudo-Rigid-Body Model was used for the compliant mechanism design, and for analysis of the large deflection flexible segments. To achieve on-chip actuation, the mechanism design was optimized to allow it to be switched using linear motion thermal actuators. The modeling theory and analysis are presented for three design iterations, with two iterations fabricated in the MUMP's process and the third in the SUMMiT process.

[795]
\end{abstract}

Index Terms-Bistable, compliant mechanism, micro relay, micro switch, pseudo-rigid-body model, thermal actuator.

\section{INTRODUCTION}

$\mathbf{M}$ ICRO-BISTABLE mechanisms have numerous potential applications, including microvalves, microswitches, relays, and micropositioners. A bistable mechanism is defined as a mechanism that has two stable positions within its range of motion. As the mechanism is moved from its first stable position to its second stable position, it passes through an unstable equilibrium position. A primary advantage of a bistable mechanism is that it only requires energy input to switch the mechanism from one state to the other, but input energy is not required to maintain the mechanism in either of the stable equilibrium positions. This behavior of the bistable mechanism is of particular importance at the microlevel when power consumption and heat dissipation are often a major concern. The advantages of increased stability, positional accuracy and low power consumption have been important driving factors in the research and development of micro-bistable devices.

Several different bistable devices and configurations have been demonstrated in the literature. Both in-plane and out-of-plane switches have been shown that utilize the snap-through effect of a fixed-fixed beam buckled under compressive residual stress [1], [2]. A thermally actuated bistable device has been demonstrated which uses a film deposited in a state of residual tension to achieve bistable motion [3]. Each of these devices requires control of the residual stress in the deposited films to achieve the bistable effect. Several bistable devices have been shown which do not rely on residual stresses [4]-[6], however these have not yet been demonstrated with a method of on-chip actuation.

Manuscript received January 4, 2002; revised April 9, 2002. This work was supported by the National Science Foundation under Grants CMS-9978737 and xyz-on-a-chip Grant DMI-9980835. Subject Editor G. B. Hocker.

The authors are with the Mechanical Engineering Department, Brigham

Young University, Provo, UT 84602 USA (e-mail: lhowell@et.byu.edu).

Digital Object Identifier 10.1109/JMEMS.2002.803284.
This paper discusses the design of an in-plane compliant bistable micromechanism with rotary motion between its two stable equilibrium positions. For successful on-chip actuation, the mechanism design has been optimized so that its displacement and force requirements fall within the allowable specification of the thermal actuators used. Because of the potential for use in autonomous systems, the power consumption of the actuators has also been reduced. Three optimized designs were fabricated and tested and the results are presented using paired thermal actuators for single pulse on-chip actuation.

\section{Compliant Mechanisms And the PSEUdO-RIGID-BODY MODEL}

A compliant mechanism derives some or all of its motion from the deflection of flexible members. The use of compliance presents several advantages including part count reduction and more precise motion. The motion of a compliant beam can be predicted using small-deflection analysis, however, as the deflection increases the linearized small-deflection equations introduce significant error. Exact solutions of large deflection problems involve elliptic integral solutions, making design synthesis difficult. The Pseudo-Rigid-Body model (PRBM) is a method of analysis that allows the large deflections to be modeled using rigid-body kinematics - greatly simplifying the design of compliant mechanisms.

Using the Pseudo-Rigid-Body model, a cantilevered beam with a force applied at the free end can be modeled as a rigid link with a pin joint and a torsional spring (see Fig. 1). By replacing the flexible segment with a rigid link and a pin-joint, the force and deflection characteristics of the member can be analyzed using rigid-body kinematics. The location of the pin joint, or characteristic pivot, is determined by the characteristic radius factor, $\gamma$. The value for $\gamma$ is a function of the angle of the applied load; however, it is reasonable to assume a value of 0.85 for a wide range of loading angles [7]. The location of the beam end can then be determined by

$$
\frac{a}{l}=\gamma(1-\cos \Theta)
$$

and

$$
\frac{b}{l}=\gamma \sin \Theta
$$

where $\Theta$ is the pseudo-rigid-body link angle, $a$ is the horizontal distance to the beam end, $b$ is the vertical distance to the beam end, and $l$ is the undeflected beam length as shown in Fig. 1(b). This method is accurate to within $0.5 \%$ for a maximum angular 


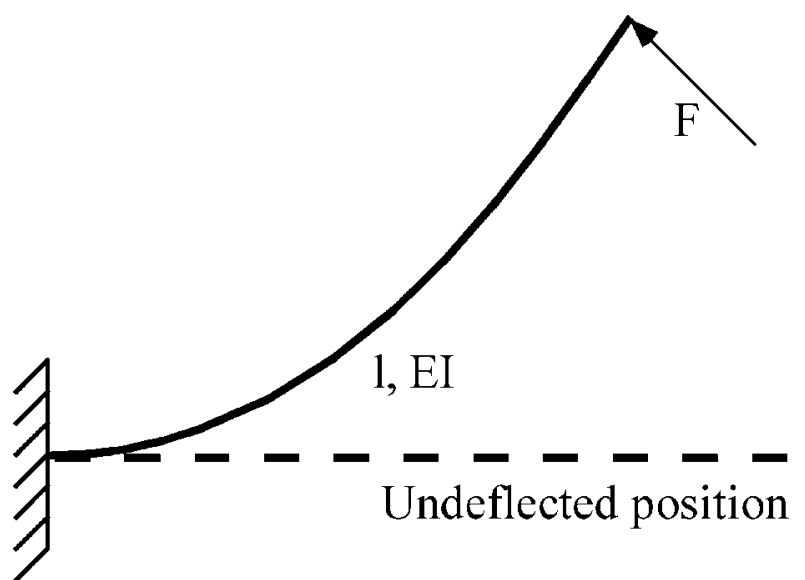

(a)

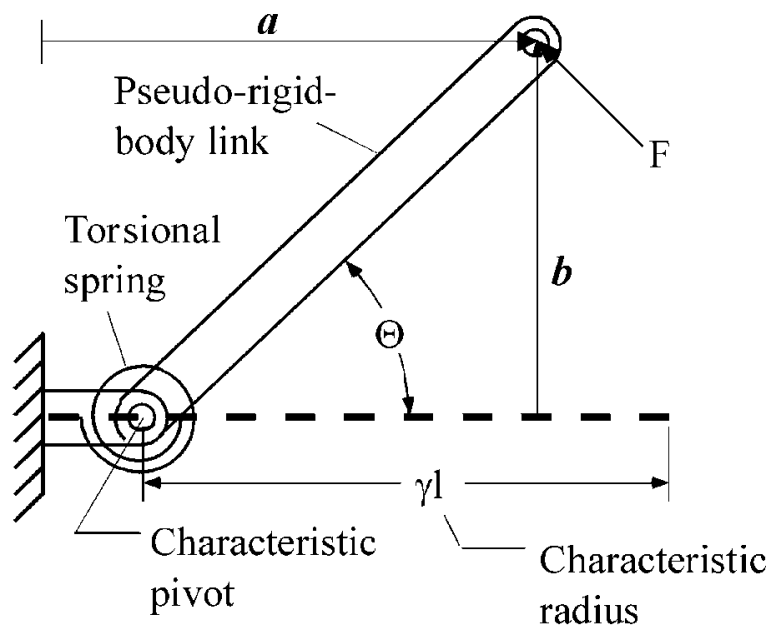

(b)

Fig. 1. (a) Original fixed-pinned segment. (b) Pseudo-Rigid-Body model of a fixed-pinned segment.

displacement of $\Theta=77^{\circ}$. A torsional spring must be added to model the resistance of the beam to bending, with the spring stiffness defined as

$$
K=\gamma K_{\theta}\left(\frac{E I}{l}\right)
$$

where $E$ is the Young's Modulus of the material, $I$ is the area moment of inertia, $l$ is the beam length and $K_{\theta}$ is the PseudoRigid-Body model stiffness coefficient, which has a value of 2.65 for most loading conditions [8]. After modeling all of the flexible segments in a device with the PRBM, the mechanism motion and energy storage can be analyzed using traditional rigid-body mechanism analysis methods. Once the geometry of a compliant mechanism is known, finite-element analysis is a useful tool for validating and fine-tuning the design, however, the PRBM is much more valuable in the early phases of design as it allows for devices to be designed that otherwise would not be feasible, with specific force and displacement characteristics.

\section{Method OF ACTUATION}

Thermal actuators are devices that utilize constrained thermal expansion of thin members to achieve an amplified motion.

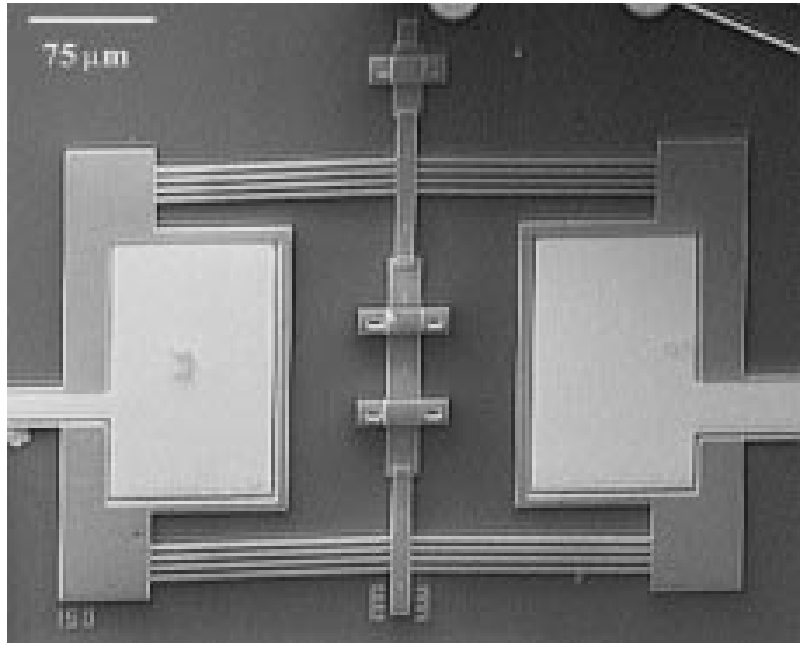

Fig. 2. SEM image of the thermal actuator design used in this work.

Several different design configurations have been proposed for thermal actuation, including the heat-drive actuator and the thermal in-plane microactuators [9], [10]. Multiple leg thermal in-plane actuators were used in this work because of the relatively large force and displacement output (see Fig. 2). Actuators based on thermal expansion offer advantages over electrostatically actuated devices because they can produce a much greater force output per unit area at significantly lower voltages. They are also capable of relatively large displacements.

The disadvantage of thermal actuators when compared to other methods is that they require a greater amount of power in their operation. The higher energy requirements are due to the energy loss from the heated legs to the surrounding air and into the substrate. However, this disadvantage can be minimized through proper actuator design. Operating in a vacuum has been shown to reduce the power requirements of the actuators used in this study by over an order of magnitude due to the absence of a medium for heat conduction from the heated legs. It has also been found that applying a rapid current pulse to the actuators, where a higher current is passed through the actuator legs for a short time interval, increases the deflection amplitude and improves the operating efficiency when compared to a steady-state operating mode [11].

\section{Bistable Mechanism Design}

The basic form of the mechanism developed in this paper consists of two thin flexible segments pinned to ground and connected to each other by a single rigid section. The mechanism consists of only a single link, but it is able to achieve its motion because of the compliance in the flexible segments. This type of mechanism has been named the "Young Mechanism" [12]. To analyze this compliant mechanism, the two fixed-pinned segments were replaced with their PRBMs, allowing the motion to be analyzed as a rigid-link four-bar mechanism with torsional springs located on each of the two pin joints that are not connected to ground (see Fig. 3). Using the following closed-form kinematics solution for a rigid-body four-bar mechanism, the 


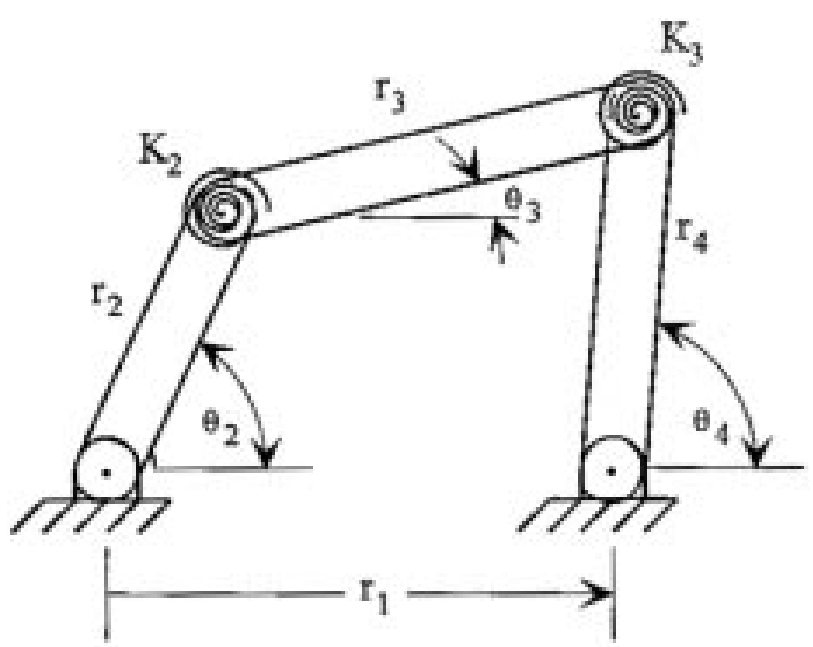

(a)

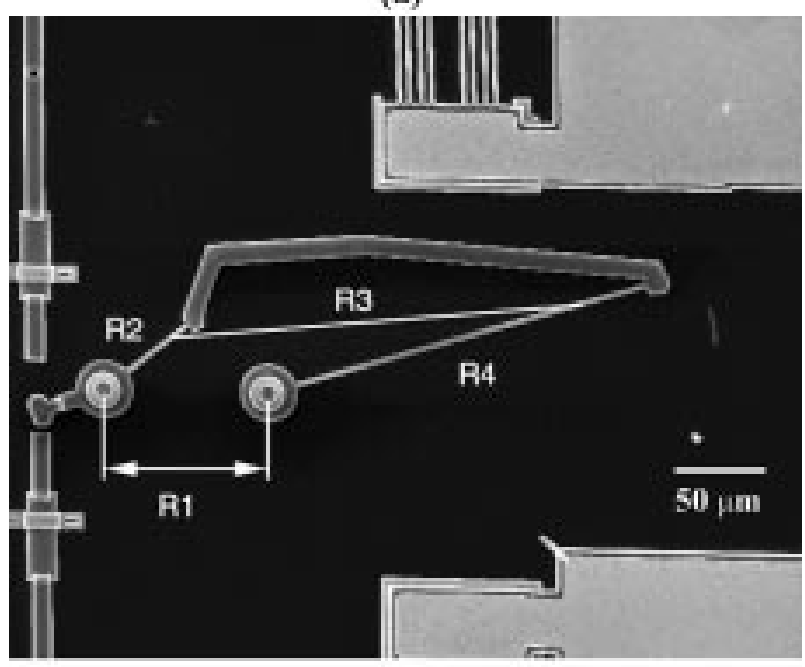

(b)

Fig. 3. (a) PRBM of bistable mechanism. (b) SEM image of mechanism with PRBM links labeled.

device motion can be calculated at any point in its rotation. For $\theta_{2}$ between 0 and $\pi$

$$
\begin{aligned}
& \theta_{3}=\psi-\beta \\
& \theta_{4}=\pi-\lambda-\beta
\end{aligned}
$$

and for $\theta_{2}$ between $\pi$ and $2 \pi$

$$
\begin{aligned}
& \theta_{3}=\psi+\beta \\
& \theta_{4}=\pi-\lambda+\beta
\end{aligned}
$$

where $\beta, \psi, \lambda$ and $\delta$ are defined as

$$
\begin{aligned}
& \beta=\cos ^{-1}\left(\frac{r_{1}^{2}+\delta^{2}-r_{2}^{2}}{2 r_{1} \delta}\right) \\
& \psi=\cos ^{-1}\left(\frac{r_{3}^{2}+\delta^{2}-r_{4}^{2}}{2 r_{3} \delta}\right) \\
& \lambda=\cos ^{-1}\left(\frac{r_{4}^{2}+\delta^{2}-r_{3}^{2}}{2 r_{4} \delta}\right)
\end{aligned}
$$

and

$$
\delta=\sqrt{r_{1}^{2}+r_{2}^{2}-2 r_{1} r_{2} \cos \left(\theta_{2}\right)}
$$

The stability of the mechanism can be determined by examining the potential energy stored in the system. For a compliant mechanism, energy is stored in the deflection of the flexible segments. The compliance in the segment is modeled, using the Pseudo-Rigid-Body model, as a torsional spring. The potential energy, $V$, of a single torsional spring is defined as

$$
V=\frac{1}{2} K\left(\theta-\theta_{i}\right)^{2}
$$

where $K$ is the torsional spring constant, $\theta$ is the link angle and $\theta_{i}$ is the initial fabricated link angle. The total energy for the mechanism is found by summing the energy stored in each of the compliant segments. For this mechanism, there are two torsional springs, and the total potential energy of the mechanism is defined as

$$
\begin{aligned}
V=\frac{1}{2} K_{2}\left[\left(\theta_{2}-\theta_{2 i}\right)\right. & \left.-\left(\theta_{3}-\theta_{3 i}\right)\right]^{2} \\
& +\frac{1}{2} K_{3}\left[\left(\theta_{4}-\theta_{4 i}\right)-\left(\theta_{3}-\theta_{3 i}\right)\right]^{2}
\end{aligned}
$$

where $K_{2}$ and $K_{3}$ are the PRBM spring constants as defined by (3), and $\theta_{2}, \theta_{3}$, and $\theta_{4}$ are the PRBM link angles. The stable and unstable equilibrium positions are then determined by plotting the total mechanism potential energy as a function of the input angle. Stable positions are located at the minimums of the potential energy curve [13], [14]. The maximum points in the potential energy curve identify the unstable equilibrium positions of the mechanism, and will be located between the stable positions. Taking the derivative of the potential energy curve with respect to the input crank angle gives the input torque required to rotate the mechanism, and is defined by

$$
\begin{aligned}
T=K_{2}[ & \left.\left(\theta_{2}-\theta_{2 i}\right)-\left(\theta_{3}-\theta_{3 i}\right)\right]\left(1-\frac{d \theta_{3}}{d \theta_{2}}\right) \\
& +K_{3}\left[\left(\theta_{4}-\theta_{4 i}\right)-\left(\theta_{3}-\theta_{3 i}\right)\right]\left(\frac{d \theta_{4}}{d \theta_{2}}-\frac{d \theta_{3}}{d \theta_{2}}\right)
\end{aligned}
$$

where

and

$$
\frac{d \theta_{3}}{d \theta_{2}}=\frac{r_{2} \sin \left(\theta_{4}-\theta_{2}\right)}{r_{3} \sin \left(\theta_{3}-\theta_{4}\right)}
$$

$$
\frac{d \theta_{4}}{d \theta_{2}}=\frac{r_{2} \sin \left(\theta_{3}-\theta_{2}\right)}{r_{4} \sin \left(\theta_{3}-\theta_{4}\right)} .
$$

The maximum stress in each of the two flexible segments can be calculated at any position in the mechanism motion using the following equations:

$$
\sigma_{2}=\frac{M c}{I}=\frac{\left\{K_{2}\left[\left(\theta_{2}-\theta_{2 i}\right)-\left(\theta_{3}-\theta_{3 i}\right)\right]\right\}\left(\frac{w_{2}}{2}\right)}{I}
$$

and

$$
\sigma_{4}=\frac{M c}{I}=\frac{\left\{K_{3}\left[\left(\theta_{4}-\theta_{4 i}\right)-\left(\theta_{3}-\theta_{3 i}\right)\right]\right\}\left(\frac{w_{4}}{2}\right)}{I}
$$

where $w$ is the width of the segment and $I$ is the cross-sectional moment of inertia.

Both the linear thermal actuator and the rotary bistable mechanism have been successfully demonstrated independently in previous works [10], [12]. However, because of the large rotations required to switch the bistable mechanism, it was not possible to actuate the device on-chip using the existing 
actuators. The thermal actuators are limited to a maximum steady-state linear displacement of approximately $12 \mu \mathrm{m}$. In order to toggle the mechanism directly with a single $12 \mu \mathrm{m}$ actuator pulse using the MUMP's surface-micromachining technology, the maximum rotation between the stable and unstable positions is limited to approximately $25^{\circ}$. This limit is based on the configuration shown in Fig. 3, where the actuator linear motion is used to flip the rotary mechanism by pushing on an extension of the mechanism input link. The minimum length of this input link extension is specified by the design rules for the fabrication process used. In addition to the displacement constraints, the input torque requirements of the mechanism are also limited by the upper bound of the actuator output force. The first design of the bistable mechanism had a required rotation of $60^{\circ}$ between the stable and unstable positions, with a total rotation of $95^{\circ}$ between the two stable positions, and as such could not be switched using a single-pulse of the actuator [12]. These devices were tested by manually displacing them with a mechanical probe. For successful on-chip actuation the bistable mechanism design must be optimized to reduce its required rotation to within the allowable output of the thermal actuators.

\section{INITIAL MECHANISM DESIGN AND VALIDATION}

These restrictions required the development of a new mechanism with small rotations that would also fall within the allowable torque restrictions. The PRBM and energy equations for the previously described mechanism [see (4)-(16)] were programmed and linked to an optimization algorithm [15] with the objective of minimizing the angle of sweep between the two stable positions by changing:

- lengths of the Pseudo-Rigid body links, $r_{1}, r_{2}$, and $r_{4}$;

- Pseudo-Rigid body link initial angles, $\theta_{2 i}$ and $\theta_{4 i}$.

Subject to the following constraintş:

- size limitation to reduce the effects of stiction and adhesion to the substrate (by restricting the size of the link $r_{3}$ to less than $300 \mu \mathrm{m})$;

- geometric check to ensure that the mechanism could be fabricated from a single layer and rotate through its motion without interference (with minimum lengths of each flexible link restricted to greater than $50 \mu \mathrm{m}$ to allow fabrication of a pin-joint);

- constraint on the magnitude of the stress in the flexible segments to prevent failure (to less than 2.0 GPa maximum stress);

- required minimum potential energy difference between unstable and stable positions to ensure that the device has enough energy to overcome stiction (with the minimum allowable potential energy difference being greater than $150 \mathrm{pJ})$.

The width of each of the two flexible links was fixed at the minimum allowed by the fabrication process. Because of the numerous local minimums in the design space, gradient-based optimization routines such as sequential quadratic programming (SQP) and generalized reduced gradient (GRG) failed to give good results; however, a simulated annealing routine provided satisfactory results and was used extensively in determining

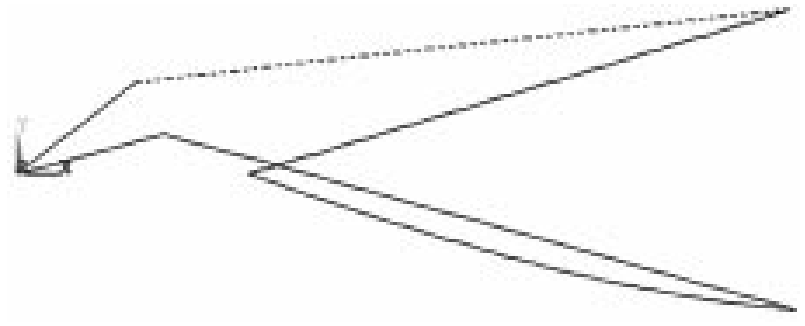

Fig. 4. ANSYS finite element predicted deformed shape at second stable position.

the optimum design [16]. The simulated annealing algorithm randomly perturbs the design variables in a search for the best design. If a better design is found, it is accepted; however, if a worse design is found, there is a probability that it will also be accepted as the new best design. This probability is based on the Boltzmann probability factor and decreases as more evaluation cycles are performed. By allowing a worse design to be accepted, the algorithm is able to jump out of local minimums in the design space in the search for a global optimum. It is this characteristic of the simulated annealing algorithm that allows it to perform well in this design problem because the design space for the young mechanism is very discontinuous with numerous local minimums.

Because of the discontinuous nature of the design space for this mechanism, and the numerous local minimums, several feasible designs were found by the optimization routine with a single design determined to be the best based on the level to which it met the optimization objective. To validate the design selected from the optimization routine, the final mechanism configuration was modeled and analyzed using a commercial finite element analysis code (ANSYS). Because of the large elastic deformations, a nonlinear analysis was performed. From the finite-element analysis, the position of the unstable equilibrium point and the second stable point were found as well as the value of the maximum stress in the mechanism at its second stable equilibrium position, and the mechanisms final deformed shape (see Fig. 4). The values obtained from finite element analysis were within $2.8 \%$ of the results predicted using the PRBM.

The final mechanism design selected from the optimization results has a total input rotation of only $23^{\circ}$ between stable positions. The potential energy and input torque curves are included in Fig. 5, and the dimensions of the mechanism are listed in Table I. The mechanism was paired with a thermal actuator in the configuration shown in Fig. 6 and was fabricated using the Multi-Layer MEMS Processes (MUMP's) at Cronos [17]. Two thermal actuators were used, with one actuator used to switch in each direction. The device proved to be bistable as predicted and was able to be actuated in either direction by a single pulse of a thermal actuator. Fig. 6 is an SEM of the device and actuators, with the mechanism shown in both its fabricated position and its second stable position. In testing done in air, the actuators required less than 8 volts and under 55 milliamps, or 440 milliwatts, to transition the device between stable positions. When tested under vacuum the power requirements were reduced by almost an order of magnitude, requiring less than 4.0 volts and under 12.0 milliamps, or a total of 48 milliwatts. This is due 


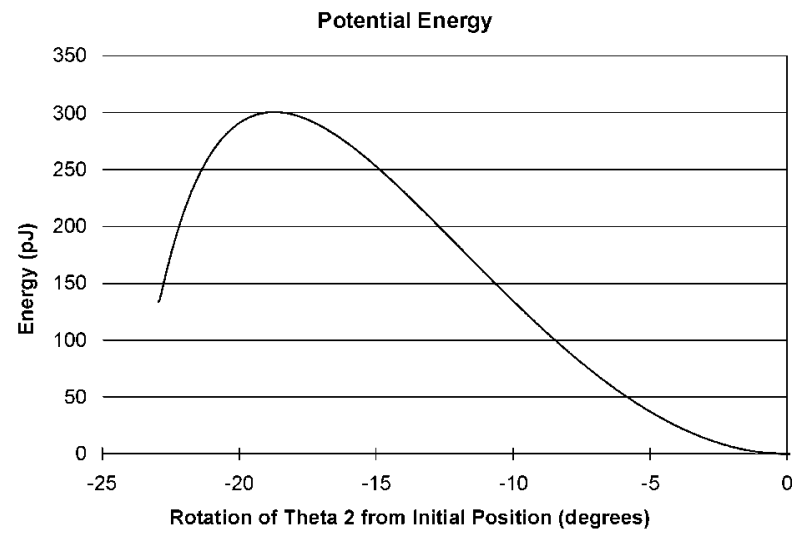

(a)

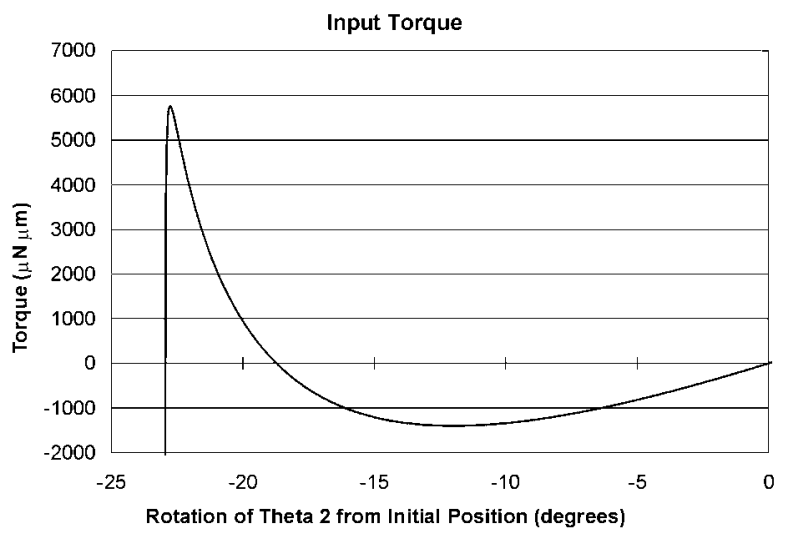

(b)

Fig. 5. (a) Potential energy and (b) input torque curves with respect to the input crank angle of the first MUMP's design iteration.

to the absence of a medium for heat loss from the legs to their surroundings.

\section{Design Refinement AND Power Reduction}

A noted disadvantage of the mechanism just described is the large difference between the required rotations to toggle the mechanism in each direction. As can be seen from the potential energy curve shown in Fig. 5, the mechanism must rotate $19^{\circ}$ from its fabricated stable position to the unstable equilibrium position, but only needs to rotate back $4^{\circ}$ to go from the second stable position to the unstable equilibrium position. The size of the thermal actuator is determined by its required force and displacement, so a much larger actuator is needed to switch the mechanism from its fabricated position. To reduce the power requirements, and achieve a more symmetric behavior, it is desirable to have the unstable equilibrium position be more centered between the two stable positions.

To achieve this, a second objective was added to the optimization setup to drive the unstable equilibrium position more toward the midpoint between the two stable positions. The widths of each of the two flexible segments were also added as design variables to increase the available design space. It was determined from the previous design that the clearance in the pin-joints introduces a significant variation in the mechanism performance. This clearance is on the order of $0.75 \mu \mathrm{m}$ and is

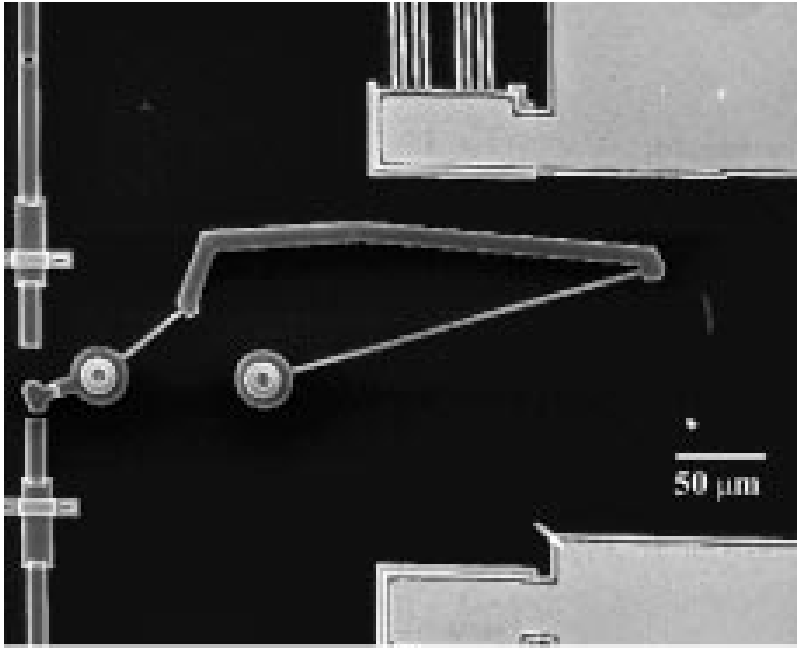

(a)

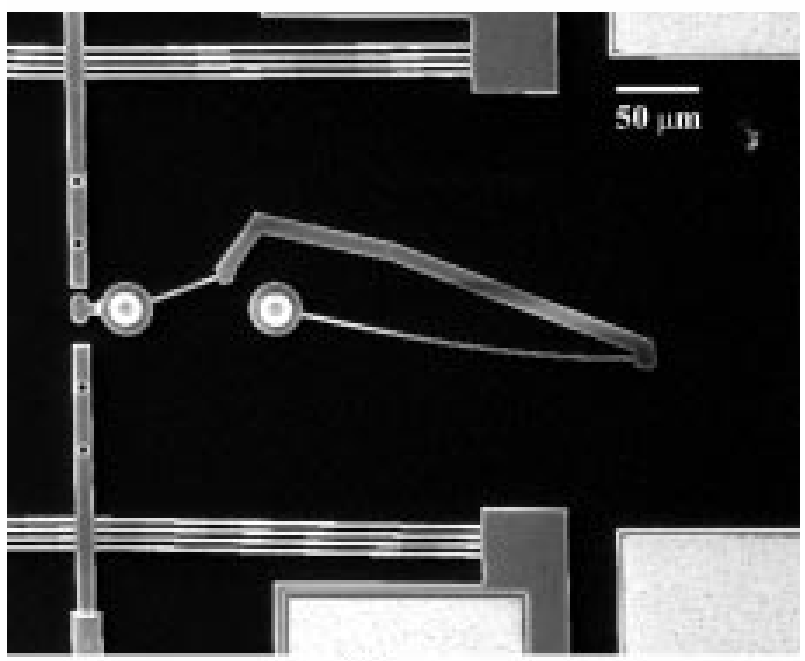

(b)

Fig. 6. SEM images of the first MUMP's iteration shown in (a) the fabricated position and (b) the second stable position (shown on two different devices).

a result of the MUMP's surface micromachining process. In an attempt to account for this variation, a tolerance on the length of the ground link of plus or minus $0.75 \mu \mathrm{m}$ was included in the optimization using a worst-case tolerance analysis. This makes the resulting design more robust to the errors introduced by the clearances.

The resulting mechanism design has a total required rotation of $23^{\circ}$ between stable positions, the same as the previous iteration. The potential energy and torque curves are included in Fig. 7, and an SEM image of the mechanism in both the fabricated position and the second stable position is shown in Fig. 8. A significant difference between this mechanism and the previous design is the increased width of the link $r_{2}$. This increase was determined by the optimization routine to improve the device function. By widening this link the torsional spring $K_{2}$ is stiffened, resulting in a more symmetric behavior. As a result, a majority of the bending occurs in the link $r_{4}$; however, the link $r_{2}$ remains flexible relative to the rigid link $r_{3}$ and continues to contribute to the mechanism performance. While similar to the previous design in total 
TABLE I

Summary of Each Young Mechanism Design

\begin{tabular}{|c|c|c|c|}
\hline Parameter & $\begin{array}{c}\text { Initial } \\
\text { MUMPs }\end{array}$ & $\begin{array}{l}\text { Refined } \\
\text { MUMPs }\end{array}$ & SUMMiT \\
\hline$r_{l}$ (microns) & 96.0 & 121.0 & 67.4 \\
\hline$r_{2}$ (microns) & 53.5 & 110.0 & 56.7 \\
\hline$r_{4}$ (microns) & 199.0 & 194.0 & 58.9 \\
\hline$\theta_{2 i}$ (degrees) & 38.0 & 11.5 & 38.7 \\
\hline$\theta_{4 i}$ (degrees) & 17.0 & -21.8 & 17.2 \\
\hline $\begin{array}{l}\text { Width of } r_{2} \\
\text { (microns) }\end{array}$ & 3.0 & 4.9 & 4.9 \\
\hline $\begin{array}{l}\text { Width of } r_{4} \\
\text { (microns) }\end{array}$ & 3.0 & 3.0 & 0.8 \\
\hline $\begin{array}{l}\text { Rotation from } \\
\text { fabricated } \\
\text { position to } \\
\text { unstable } \\
\text { equilibrium } \\
\text { (degrees) }\end{array}$ & 18.7 & 9.3 & 9.9 \\
\hline $\begin{array}{l}\text { Rotation from } \\
\text { second stable } \\
\text { position to } \\
\text { unstable } \\
\text { equilibrium } \\
\text { (degrees) }\end{array}$ & 4.2 & 14.1 & 6.0 \\
\hline $\begin{array}{l}\text { Max Stress } \\
(\mathrm{GPa})\end{array}$ & 1.22 & 0.92 & 0.70 \\
\hline $\begin{array}{c}\text { Minimum } \\
\text { potential } \\
\text { energy } \\
\text { difference }(\mathrm{pJ})\end{array}$ & 167 & 233 & 155 \\
\hline
\end{tabular}

rotation, it is more symmetric about the unstable equilibrium point, with $9^{\circ}$ of rotation from the fabricated position to the unstable position, and $14^{\circ}$ from the unstable position to the second stable position. This gives it a maximum required rotation of $14^{\circ}$, compared to $19^{\circ}$ for the previous mechanism. The peak torque values for the second iteration are similar in magnitude to the previous design, but the input torque curve has a smaller gradient so better performance is expected. This mechanism configuration was also fabricated using the MUMP's process and was successfully actuated in each direction using thermal actuators. Because of its lower maximum required angular displacement, smaller thermal actuators could be used, resulting in lower power requirements; however, for the initial fabrication the same actuators were used. Because of this, testing of this mechanism resulted in similar current and voltage requirements as for the previous iteration. Both design iterations are summarized in Table I.

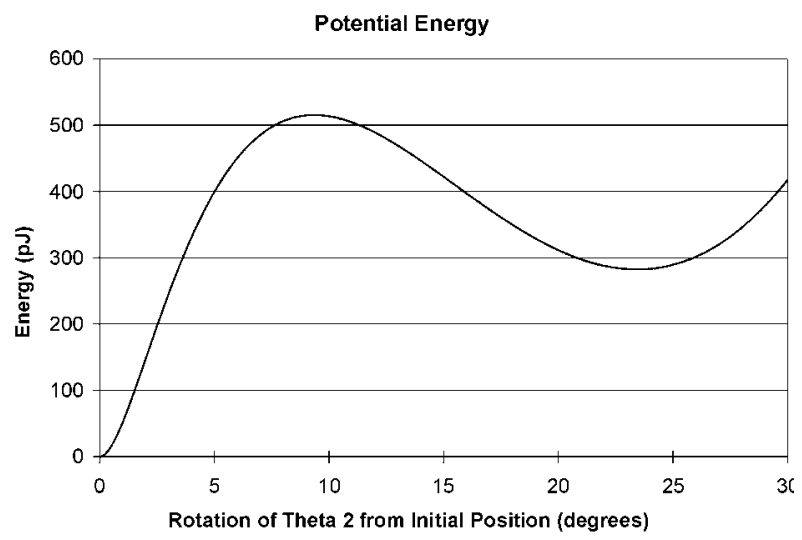

(a)

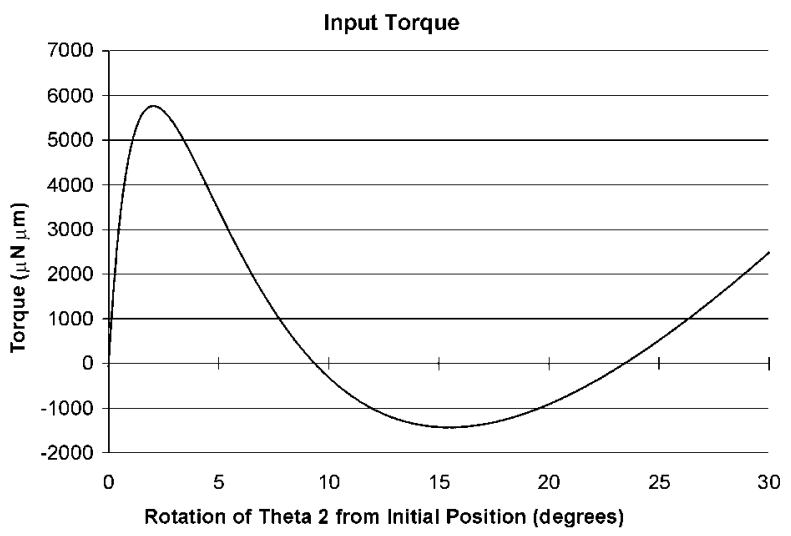

(b)

Fig. 7. (a) Potential energy and (b) input torque curves with respect to the input crank angle of the second MUMP's design iteration.

\section{SANDIA SUMMIT V DESIGN OPTIMIZATION}

Both of the previous two designs were optimized for and fabricated in the MUMP's process, which has a minimum line width of $3 \mu \mathrm{m}$, and a pin-joint clearance of plus or minus $0.75 \mu \mathrm{m}$. Because these two process constraints significantly affect the performance and size of the Young mechanism, it would be advantageous to reduce these processing parameters. The Sandia SUMMiT V process allows for $0.8 \mu \mathrm{m}$ minimum line widths, and has a specialized pin-joint cut that creates pin-joints with less than $0.3 \mu \mathrm{m}$ clearance. These two advantages alone make the SUMMiT process much more favorable for the design of Young mechanisms.

The optimization routine described in the previous section was modified slightly to allow for the smaller minimum line width and pin-joint clearance, and the device was reoptimized for the SUMMiT process. The resulting potential energy and torque curves are shown in Fig. 9, with the resulting geometry listed in Table I, along with the previous two MUMP's designs for comparison. SEM images of the mechanism in both positions are included in Fig. 10. Because of the availability of additional planarized layers in the SUMMiT process, a bar was added across the mechanism to prevent any motion out of the plane of the device. The addition of this feature is not possible using the MUMP's process; however, the additional layers in the SUMMiT process are not required for the Young mechanism. 


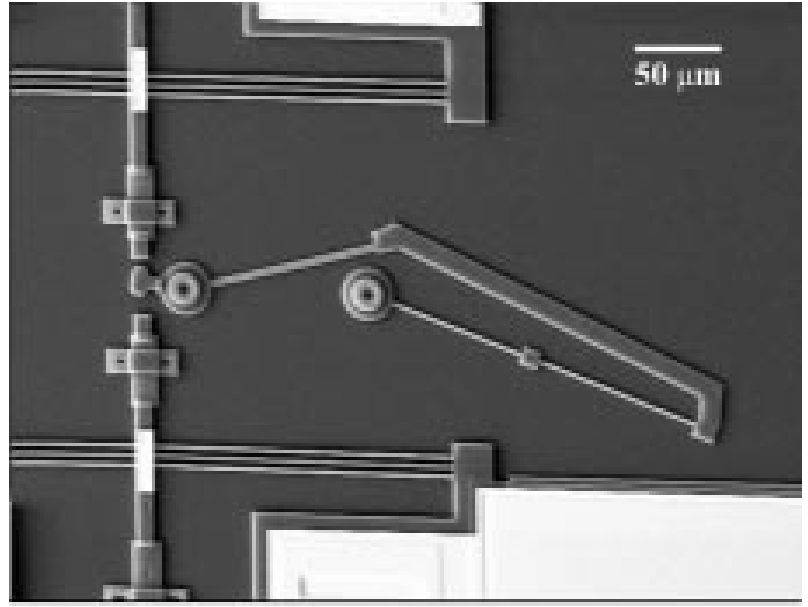

(a)

Fig. 8. SEM images of the second MUMP's iteration shown in (a) the fabricated position and (b) second stable position (shown on two different devices).

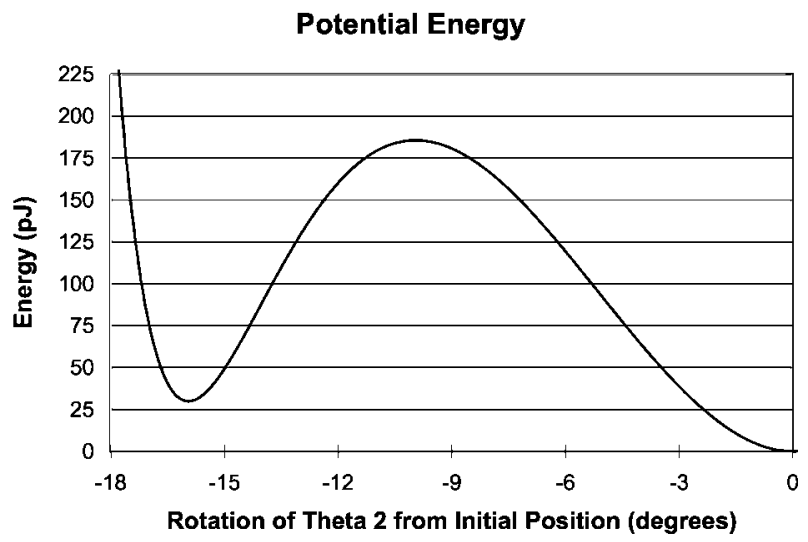

(a)

Input Torque

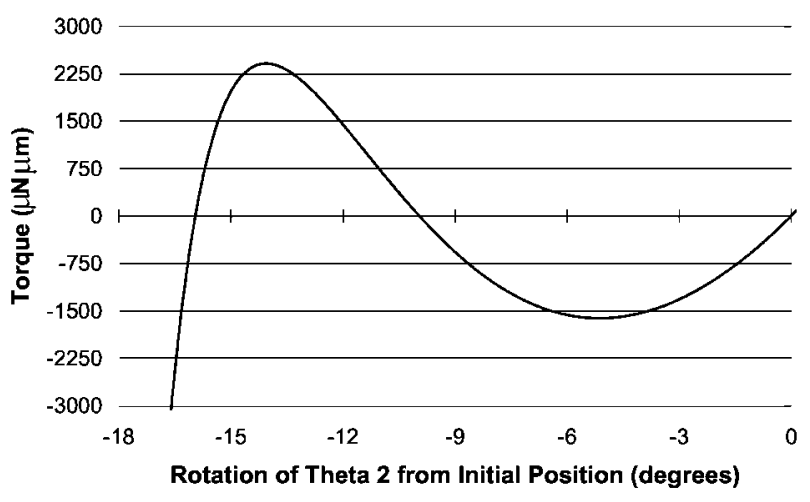

(b)

Fig. 9. (a) Potential energy and (b) input torque curves with respect to the input crank angle for the SUMMiT design.

This design was successfully tested with the lowest power requirements of any of the three designs, requiring only 3.0 volts and 11.0 milliamps in air, or a total of 33 milliwatts. This design resulted in a lower power requirement in air than the previous two iterations required in vacuum. Much of this improvement is due to design changes in the thermal actuators that were only

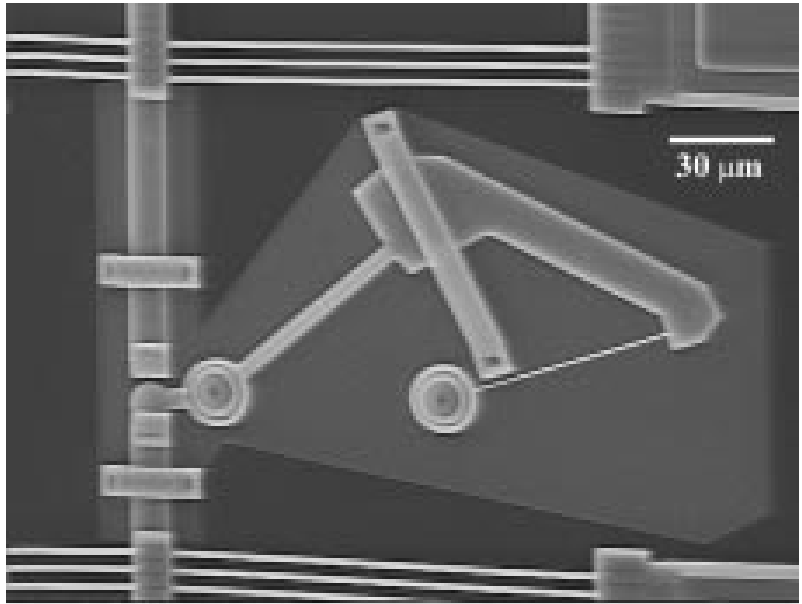

(a)

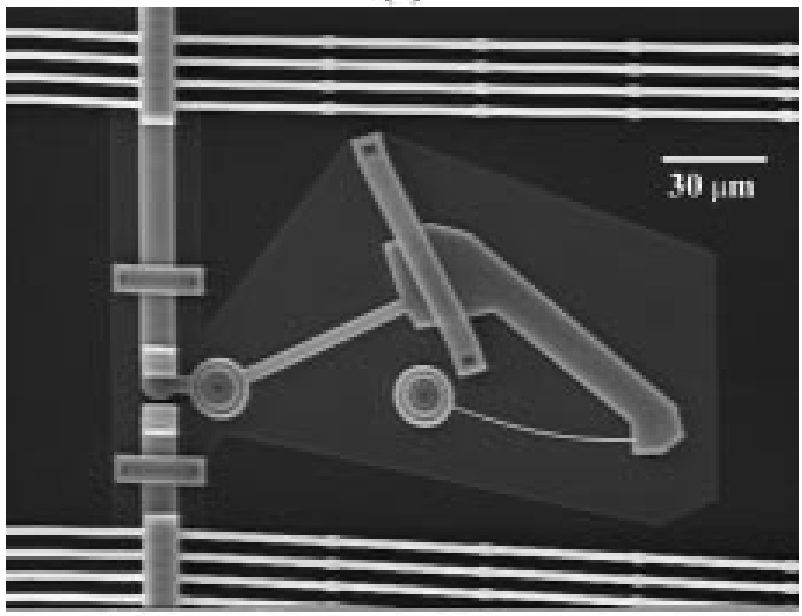

(b)

Fig. 10. SEM images of the SUMMiT design shown in (a) the fabricated position and (b) the second stable position (shown on two different devices).

possible in the SUMMiT process, such as elevating the heated legs higher off the substrate using the Poly3 and Poly4 layers. This increased gap decreases the heat loss to the substrate, increasing the efficiency of the actuator. In addition, significant savings were realized because the actuators could be smaller due to the reduced force and displacement requirements of the SUMMiT Young mechanism design.

\section{CONCLUSION}

By combining an accurate large-deflection model of a bistable mechanism configuration with an optimization algorithm, a device has been developed which can be actuated on-chip using linear thermal actuators. Three successful design iterations have been demonstrated, two having been fabricated and tested using the MUMP's surface micromachining technology, and one in the SUMMiT process. Each configuration can be switched in either direction with a single pulse of a thermal actuator. By minimizing the required displacement of the actuators their size has been reduced, lowering the power requirements from previous design iterations. The smaller line-widths and geometries that are possible in the SUMMiT process have proven to be a significant advantage in the design 
of both the thermal actuator and the Young mechanism, allowing the smallest mechanism with the lowest actuator power requirements.

\section{ACKNOWLEDGMENT}

The authors gratefully acknowledge the assistance of $\mathrm{J}$. Wittwer.

\section{REFERENCES}

[1] B. Hälg, "On a nonvolatile memory cell based on microelectromechanics," J. Microelectromech. Syst., pp. 172-176, 1990.

[2] M. Vangbo and Y. Bäcklund, "A lateral symmetrically bistable buckled beam," J. Micromechan. Microeng., vol. 8, pp. 29-32, 1998.

[3] H. Matoba, T. Ishikawa, C. Kim, and R. S. Muller, "A bistable snapping mechanism," IEEE Micro Electro Mechanical Systems 1994, pp. 45-50, 1994.

[4] E. E. J. Kruglick and K. S. J. Pister, "Bistable mems relays and contact characterization," in Solid-State Sensor and Actuator Workshop, Hilton Head Island, SC, June 8-11, 1998, pp. 333-337.

[5] M. B. Parkinson, B. D. Jensen, and G. M. Roach, "Optimization-based design of a fully-compliant bistable micromechanism," in Proc. 2000 ASME Design Engineering Technical Conf., Baltimore, MD, Sept. $10-13,2000$.

[6] M. S. Baker, S. M. Lyon, and L. L. Howell, "A linear displacement bistable micromechanism," in Proc. 2000 ASME Design Engineering Technical Conf., Baltimore, MD, Sept. 10-13, 2000.

[7] L. L. Howell and A. Midha, "Parametric deflection approximations for end-loaded, large-deflection beams in compliant mechanisms," ASME J. Mechan. Design, vol. 117, no. 1, pp. 156-165, 1995.

[8] L. L. Howell, Compliant Mechanisms. New York: Wiley, 2001.

[9] J. H. Comtois, M. A. Michalicek, and C. C. Barron, "Electrothermal actuators fabricated in four-level planarized surface micromachined polycrystalline silicon," Sens. Actuators, Phys. A, vol. 70, pp. 23-31, 1998.

[10] R. Cragun and L. L. Howell, "Linear thermomechanical microactuators," in Microelctromechanical Systems (MEMS), at the 1999 ASME Int. Mechanical Engineering Congress and Exposition, November 1999, pp. 181-188.

[11] C. D. Lott, "Electrothermomechanical modeling of a surface micromachined linear displacement microactuator," Master's thesis, Brigham Young University, Aug. 2001.

[12] B. D. Jensen, L. L. Howell, and L. G. Salmon, "Design of two-link in-plane, bistable compliant micro-mechanisms," ASME J. Mechan. Design, vol. 121, pp. 416-423, Sept. 1999.
[13] P. G. Opdahl, B. J. Jensen, and L. L. Howell, "An investigation into compliant bistable mechanisms," in Proc. 1998 ASME Design Engineering Technical Conf., Atlanta, GA, Sept. 13-16, 1998.

[14] G. J. Simitses, An Introduction to the Elastic Stability of Structures. Englewood Cliffs, NJ: Prentice-Hall Inc., 1976.

[15] A. Parkinson, R. Balling, and J. Free, "OptdesX: An X window-based optimal design software system," in Proc. Fourth AIAA/USAF/NASA/OAI Symp. Multidisciplinary Analysis and Optimization, Cleveland, OH, Sept. 21-23, 1992, p. 559.

[16] S. Kirkpatrick, C. D. Gelatt, and M. P. Vecchi, "Optimization by simulated annealing," Science, vol. 220, no. 4598, p. 671, 1983.

[17] D. A. Koester, R. Mahadevan, A. Shishkoff, and K. W. Markus, MUMPS Design Handbook: Cronos Integrated Microsystems, 1999.

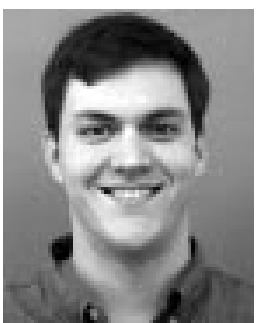

Michael S. Baker received the M.S. degree in mechanical engineering from Brigham Young University (BYU), Provo, UT, in 2002. His research efforts were in the area of MEMS bistable mechanisms and actuation.

He is currently a Member of the Technical Staff in the MEMS Device Concepts Department at Sandia National Laboratories, Albuquerque, NM. His research interests include compliant mechanism design and methods of actuation in MEMS.

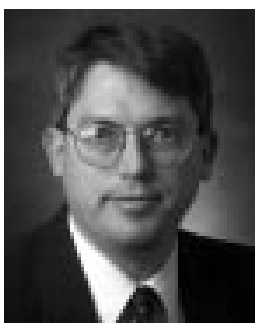

Larry L. Howell received the B.S. degree from Brigham Young University (BYU), Provo, UT, in 1987 and the M.S. and Ph.D. degrees from Purdue University, West Lafayette, IN, in 1991 and 1993, respectively. He is currently Chair of the Department of Mechanical Engineering at BYU. Prior to joining BYU in 1994, he was a Visiting Professor at Purdue University, a finite-element analysis consultant for Engineering Methods, Inc., and an Engineer on the design of the YF-22 (the prototype for the U.S. Air Force F-22). He is a Director of the Utah Center of Excellence in Compliant Mechanisms and has over ten patents granted or pending in the area of MEMS and compliant mechanisms. He is the author of the book Compliant Mechanisms (New York: Wiley)

Dr. Howell is the recipient of an NSF CAREER Award (1996-2000), the ASME Design Automation Conference Best Paper Award (1999), the MDI Software Award (1992), and he is a member of the ASME Mechanisms Committee. 